BUHEP 95-3

hep-ph/9502267

February 9, 1995

\title{
The profile of a nonstandard Higgs boson at the $\mathrm{LHC}$
}

\author{
Dimitris Kominis and Vassilis Koulovassilopoulosi \\ Boston University, Physics Department, \\ 590 Commonwealth Avenue, \\ Boston, MA 02215 USA
}

\begin{abstract}
In a wide class of extensions of the Standard Model there is a scalar resonance with the quantum numbers of the usual Higgs boson but with different couplings to fermions and gauge bosons. Using an effective Lagrangian description, we examine the phenomenology of such a generic nonstandard Higgs boson at the LHC. In particular, we determine the circumstances under which such a particle can be observed in its $Z Z$ decay mode and distinguished from the Higgs boson of the Standard Model. We briefly comment on the energy scale effectively probed at the LHC, if the nonstandard nature of an observed Higgs particle can be asserted.
\end{abstract}

*e-mail address: kominis@budoe.bu.edu

$\dagger$ e-mail address: vk@budoe.bu.edu 


\section{Introduction}

The operation of the next generation of high-energy colliders (such as the LHC, LEP-II, NLC) within the coming decade is expected to bring us closer to an understanding of the mechanism of electroweak symmetry breaking. The minimal Standard Model (SM) is the simplest possibility, but its confirmation requires the discovery of a neutral scalar particle, the Higgs boson, with properties completely specified once given its mass. In the SM this is an undetermined parameter, and so far direct searches have set a lower limit of about $60 \mathrm{GeV}$ [1]. An upper bound of approximately $1 \mathrm{TeV}$ has been suggested on the basis of "triviality" [2], and the validity of the perturbation expansion [3], which makes it likely that, if the SM Higgs boson exists, it will be discovered at the next generation colliders.

However, it is widely believed [2, [4] that the SM, despite its experimental success, can not be complete and that new physics, beyond the SM, should arise at some finite energy scale $\Lambda$. If $\Lambda$ is very large, then the low-energy theory would look like the SM, while if $\Lambda$ is low (such as a few $\mathrm{TeV}$ ), then deviations should be expected and the properties of a Higgs-like resonance (if present) could differ substantially from those predicted in the context of the SM. A resonance lighter than other massive degrees of freedom that shares the quantum numbers of the SM Higgs boson but couples to the electroweak gauge bosons and to fermions with nonstandard strength has been generically called a "Nonstandard Higgs" boson [5.6].

Such an object is featured in a variety of models of electroweak symmetry breaking; namely, some models with dynamical symmetry breaking, such as Composite Higgs models [7.8.9.10,6] and "top-condensate" models [11], as well as linear models with many fundamental scalars in which a mass gap exists between a light scalar-isoscalar (under custodial isospin) particle and all other resonances. If these models describe electroweak symmetry breaking, the isoscalar resonance presumably will be the first to be discovered in a collider experiment. It is not clear a priori, however, whether its nonstandard properties can be measured accurately enough to distinguish it from the SM Higgs boson.

The question we wish to address in this paper is whether it will be possible in future experiments at the Large Hadron Collider (LHC) to detect a nonstandard Higgs boson and to differentiate it from the SM Higgs. As a model, we consider the most general low-energy effective Lagrangian in terms of the usual $S U(2)_{L} \times S U(2)_{R} / S U(2)_{V}$ symmetry breaking

pattern which, below the cutoff scale $\Lambda$, has the same spectrum as the SM. The SM is 
a particular case and corresponds to the limit where $\Lambda \rightarrow \infty$. This Lagrangian is then used to explore the prospects of the LHC to detect and distinguish a nonstandard from a Standard Higgs boson. In particular, for a variety of Higgs boson masses $m_{H}$ (assuming that $m_{H}>2 m_{Z}$ ), we determine the values of couplings in the effective Lagrangian for which this is possible by looking at the Higgs boson decay mode $H \rightarrow Z Z \rightarrow l^{+} l^{-} l^{+} l^{-}$, where $l$ is an electron or a muon. It has been shown [5,6] that if a scalar isoscalar resonance is observed, then the measurement of its width offers the best way to distinguish it from the SM Higgs.

The deviations from the SM couplings can be used within specific models to estimate the scale $\Lambda$ of new physics, provided no other nonstandard physics is discovered. As an indication, we have done so for a number of simple Composite Higgs models. This is similar in spirit to an early study by Kosower [10], who also proposed the measurement of the width as a tool to probe the compositeness scale within Composite Higgs models. However, we performed a more detailed statistical analysis and reached somewhat different (less optimistic) conclusions.

In the next section we review the theoretical framework and construct the effective Lagrangian of the most general theory with a nonstandard Higgs boson. In Section 3 we describe the calculation of the signal and $Z Z$ background cross-sections and discuss the issue of whether one can discriminate between a nonstandard Higgs boson and its SM counterpart on the basis of a width measurement. In particular, we derive the statistical significance of a possible discrepancy between the result of such a measurement and the SM expectation. Finally, Section 4 contains our conclusions.

\section{The Effective Lagrangian}

In this section, we briefly describe the construction of the most general effective theory with a nonstandard Higgs boson [5,6]. The electroweak symmetry breaking sector at low energies contains, besides the Goldstone bosons $w^{a}$ (which become the longitudinal components of $W^{ \pm}$and $Z$ ), one extra scalar particle $H$ (the nonstandard Higgs boson) with the quantum numbers of the SM Higgs boson円.

As in the SM, we assume that the Goldstone bosons arise from the spontaneous break-

\footnotetext{
${ }^{1}$ Throughout, we shall call a generic nonstandard Higgs boson simply a Higgs boson unless otherwise stated.
} 
down of a chiral $S U(2)_{L} \times S U(2)_{R}$ symmetry down to its diagonal $S U(2)_{V}$ subgroup. As usual, $S U(2)_{L}$ is identified with the gauge group $S U(2)_{W}$ and $S U(2)_{R}$ is the "custodial" symmetry whose $\tau_{3}$ component is identified with hypercharge. The interactions of the Goldstone bosons are described conveniently by using a nonlinear realization [12] of the chiral symmetry, in terms of the field

$$
\Sigma=\exp \left(\frac{i \vec{w} \cdot \vec{\tau}}{v}\right)
$$

where $\vec{\tau}$ are the Pauli matrices corresponding to the broken $S U(2)$ generators, normalized so that $\operatorname{Tr}\left(\tau^{a} \tau^{b}\right)=2 \delta^{a b}$, and $v=246 \mathrm{GeV}$ is the weak scale. Under the $S U(2)_{L} \times S U(2)_{R}$ chiral symmetry the fields $(\Sigma, H)$ transform as

$$
\Sigma \rightarrow L \Sigma R^{\dagger} \quad, \quad H \rightarrow H
$$

where $L$ and $R$ are $S U(2)_{L}$ and $S U(2)_{R}$ matrices respectively. Then, the most general chirally invariant Lagrangian that describes the interactions of the isoscalar $H$ with the Goldstone bosons $w^{a}$, to lowest order in momentum, can be written as

$$
\mathcal{L}=\frac{1}{4}\left(v^{2}+2 \xi v H+\xi^{\prime} H^{2}+\ldots\right) \operatorname{Tr}\left(\partial_{\mu} \Sigma^{\dagger} \partial^{\mu} \Sigma\right)+\mathcal{L}_{H}
$$

where $\mathcal{L}_{H}$ is the Lagrangian that describes the Higgs boson self-interactions

$$
\mathcal{L}_{H}=\frac{1}{2}\left(\partial_{\mu} H\right)^{2}-\frac{m_{H}^{2}}{2} H^{2}-\frac{\lambda_{3} v}{3 !} H^{3}-\frac{\lambda_{4}}{4 !} H^{4}-\ldots
$$

and $\xi, \xi^{\prime}, \lambda_{3}$ and $\lambda_{4}$ are unknown coefficients. For simplicity, in eqs. (3) and (4) we only show the leading terms, with the ellipsis denoting higher powers in $H$.

The gauge bosons can be introduced by replacing the ordinary derivative in eq. (3) by the covariant one, which, by virtue of the transformation law (2), takes the form

$$
D_{\mu} \Sigma=\partial_{\mu} \Sigma+i \frac{g}{2} \vec{\tau} \cdot \vec{W}_{\mu} \Sigma-i \frac{g^{\prime}}{2} B_{\mu} \Sigma \tau_{3}
$$

where $g, g^{\prime}$ are the usual $S U(2)_{W}$ and $U(1)_{Y}$ gauge couplings respectively. Hence the parameters $\xi, \xi^{\prime}$ etc, represent the couplings of one or more nonstandard Higgs bosons to a pair of weak gauge bosons $W_{\mu}^{a}$. 
The fermions are incorporated into the effective Lagrangian as matter fields [12]. We shall only consider the quarks of the third family, since these will be the only important ones in our phenomenological investigation. These fermions can be included in the fields

$$
\psi_{L}=\left(\begin{array}{c}
t_{L} \\
b_{L}
\end{array}\right) \quad, \quad \psi_{R}=\left(\begin{array}{c}
t_{R} \\
b_{R}
\end{array}\right)
$$

which transform as $\psi_{L} \rightarrow L \psi_{L}$ and $\psi_{R} \rightarrow R \psi_{R}$ under $S U(2)_{L} \times S U(2)_{R}$. Their interactions with the scalars are given by

$$
\mathcal{L}_{\Sigma f \bar{f}}=h_{1}\left(v+y_{1} H+\ldots\right) \bar{\psi}_{L} \Sigma \psi_{R}+h_{2}\left(v+y_{2} H+\ldots\right) \bar{\psi}_{L} \Sigma \tau_{3} \psi_{R}+\text { h.c. }
$$

where $h_{1}$ and $h_{2}$ correspond to Yukawa couplings and can be replaced by the fermion masses, through $m_{t}=\left(h_{1}+h_{2}\right) v$ and $m_{b}=\left(h_{1}-h_{2}\right) v$, while $y_{1}$ and $y_{2}$ are new unknown couplings. Again, the ellipsis denotes higher powers in the Higgs field which are not included in our analysis. Using the explicit form (6) in eq. (7), we can read off the Higgs boson couplings to the top and bottom quarks:

$$
\begin{aligned}
\mathcal{L}_{H f \bar{f}} & =\left(h_{1} y_{1}+h_{2} y_{2}\right) H \bar{t}_{L} t_{R}+\left(h_{1} y_{1}-h_{2} y_{2}\right) H \bar{b}_{L} b_{R}+\text { h.c. } \\
& \equiv y_{t}\left(m_{t} / v\right) H \bar{t}_{L} t_{R}+y_{b}\left(m_{b} / v\right) H \bar{b}_{L} b_{R}+\text { h.c. }
\end{aligned}
$$

Thus, this Lagrangian introduces two new unknown parameters $y_{t}, y_{b}$.

The SM is a particular case of the effective theory defined above, with the only nonzero couplings being

$$
\xi, \xi^{\prime}=1, \lambda_{3}, \lambda_{4}=\frac{3 m_{H}^{2}}{v^{2}} \quad, \quad y_{t}, y_{b}=1
$$

For values of the couplings different from (9) the effective Lagrangian is nonrenormalizable and a cutoff is implicitly present. We may estimate the order of magnitude of this cutoff by determining the scale at which partial wave unitarity is violated. The isospin-0 spin-0 partial wave amplitude, for $s \gg m^{2}$, is [5, 13.

$$
a_{00}(s)=\left(1-\xi^{2}\right) \frac{s}{16 \pi v^{2}}
$$

At a scale

$$
s=\frac{16 \pi v^{2}}{\left|1-\xi^{2}\right|}
$$


partial wave unitarity breaks down. Consequently the cutoff $\Lambda$ of the theory, physically associated with the scale at which new degrees of freedom emerge, must lie at or below this scale. From eq. (11) it becomes clear that the larger the deviation of $\xi$ from its SM value of 1 , the lower the energy scale at which new physics is expected. If $\Lambda \equiv 4 \pi f$ is the scale of new physics, then by inverting eq. (11), we can write, roughly,

$$
\xi^{2}=1+O\left(\frac{v^{2}}{f^{2}}\right)
$$

This is effectively the statement that nonrenormalizable couplings must be suppressed by powers of the scale of new physics. We thus expect similar relations to hold for the other couplings $y_{t}, y_{b}, \xi^{\prime}$, etc. Relations such as (12) are borne out by calculations in specific models [0, 8,9, 10,6]. Higher momentum contributions to the effective action can be systematically taken into account using chiral perturbation theory [14], although, for simplicity, we do not include them in this study.

It is clear that, since a nonstandard Higgs boson couples to the same channels as its SM counterpart, its search strategy will be based on the same signatures. Here, we shall assume that $m_{H}>2 m_{Z}$. In this mass range, it turns out that the nonstandard Higgs boson properties are determined, to lowest order in perturbation theory, by only two parameters, namely $\xi$ and $y_{t}$. The Higgs boson decay width, for example, is given by

$$
\begin{aligned}
\Gamma_{H}= & \frac{m_{H}^{3}}{32 \pi v^{2}} \xi^{2}\left[2 \sqrt{1-x_{W}}\left(1-x_{W}+\frac{3}{4} x_{W}^{2}\right)+\sqrt{1-x_{Z}}\left(1-x_{Z}+\frac{3}{4} x_{Z}^{2}\right)\right] \\
& +\frac{3 m_{t}^{2} m_{H}}{8 \pi v^{2}} y_{t}^{2}\left(1-\frac{4 m_{t}^{2}}{m_{H}^{2}}\right)^{3 / 2} .
\end{aligned}
$$

where $x_{V}=4 m_{V}^{2} / m_{H}^{2}, V=W, Z$. Here we are assuming that the underlying shortdistance dynamics acts so as not to particularly enhance $y_{b}$ over $y_{t}$. Then, since $m_{t} \gg m_{b}$, only the top quark couples significantly to the Higgs boson $H$, and we can ignore the coupling to the bottom quark. On the other hand, in the purely scalar sector, tree level amplitudes do not depend on the parameters $\xi^{\prime}, \lambda_{3}, \lambda_{4}$, etc. The leading one-loop corrections to $W_{L} W_{L}$ scattering and the Higgs boson decay width were computed in Ref. [5.6] and, for phenomenological purposes, they can be incorporated in the effective definition of $\xi$. We now proceed to investigate the phenomenology of the model presented above. 


\section{Phenomenology}

In this section, we explore the phenomenology of a nonstandard Higgs boson at the LHC. We shall only consider Higgs boson masses larger than the $Z Z$ threshold. In this mass range, the Higgs boson decays primarily to gauge boson pairs and thus can be most effectively searched for in the "gold-plated" channel

$$
H \rightarrow Z Z \rightarrow l^{+} l^{-} l^{+} l^{-}
$$

where $l$ is an electron or a muon. This process has been discussed at great length in the literature ${ }^{2}$, and it is expected that a Standard Higgs boson with mass $m_{H} \leq 500 \mathrm{GeV}$ $(800 \mathrm{GeV})$ will be discovered at the LHC at an integrated luminosity of $10 \mathrm{fb}^{-1}\left(100 \mathrm{fb}^{-1}\right)$. The main question we try to answer in the analysis that follows is whether a nonstandard Higgs boson of given mass $m_{H}$ and couplings $\xi$ and $y_{t}$ can be detected at the LHC and distinguished from a SM Higgs boson of the same mass. We present results for integrated luminosities of $10 \mathrm{fb}^{-1}$ and $100 \mathrm{fb}^{-1}$, while the center-of-mass energy is assumed to be $\sqrt{s}=14.6 \mathrm{TeV}$. In our estimates of cross-sections we have used the ELHQ structure functions, set 2 [18].

The main background to the four lepton signal (14) comes from the Born process

$$
q \bar{q} \rightarrow Z Z \rightarrow l^{+} l^{-} l^{+} l^{-}
$$

We calculated this background imposing the following cuts on the rapidity and transverse momentum of the $Z$ bosons

$$
\left|\eta^{Z}\right|<2 \quad ; \quad p_{T}^{Z}>\frac{1}{4} \sqrt{m_{Z Z}^{2}-4 m_{Z}^{2}} .
$$

The rapidity cut for the $Z$ 's translates, approximately, into a cut of 2.5 for the rapidities of the final state leptons. We included part of the QCD corrections to this process, through a "K-factor" 19

$$
K=1+\frac{8}{9} \pi \alpha_{s}\left(m_{H}\right)
$$

An irreducible $Z Z$ background also arises from gluon fusion through a quark loop (the 'box' diagram)

$$
g g \rightarrow Z Z .
$$

\footnotetext{
${ }^{2}$ Recent reviews appear, for example, in Refs. 15, 16, 17.
} 
In fact, this process interferes with the resonant Higgs boson exchange process

$$
g g \rightarrow H \rightarrow Z Z
$$

where the Higgs boson is produced through a top quark loop. An exact calculation for the SM [20] has shown that the effect of the interference is rather small, for most of the range of masses we consider. Towards the upper end of this range, however, (that is $m_{H} \approx 800 \mathrm{GeV}$ ) the increase in the cross-section caused by the interference term may become sizeable (it is constructive interference). We ignored this effect, and thus our estimates of the signal rate are somewhat conservative for large masses. We did take into account, however, the contribution of the 'box' diagram to the background, which amounts to approximately $50 \%$ of the Born process (15), by scaling the cross-section of the latter by 1.5 .

There are also reducible four-lepton backgrounds, primarily from $t \bar{t}$ production. It has been argued [15,21] that, with appropriate isolation cuts and the expected $Z$ mass resolution capability at the LHC, these backgrounds can be reduced to well below the irreducible background levels. We shall therefore ignore them in this study. However, we have taken into account a $10 \%$ loss of signal rate due to these cuts 21.

The main mechanism for $Z$-boson pair production through a Higgs boson is the process (19). The rate for this process depends on the top quark mass, which is chosen here to be $m_{t}=170 \mathrm{GeV}$ [22], and also on the nonstandard Yukawa coupling $y_{t}$. In the Standard Model, for such a top-quark mass, the gluon fusion is the dominant production mechanism for all Higgs boson masses up to $1 \mathrm{TeV}$. Leading QCD corrections to this process have been included by multiplying the cross section by another "K-factor" [23,24, 25]

$$
K=1+\left(\frac{11}{2}+\pi^{2}\right) \frac{\alpha_{s}\left(m_{H}\right)}{\pi} .
$$

A second production mechanism for $Z$ pairs through a Higgs boson is gauge boson fusion

$$
q q \rightarrow q q H \rightarrow q q Z Z
$$

We computed the cross-section for this process by using the effective- $W$ approximation 26,27. The scattering amplitudes are calculated at tree-level in the gauge theory from the Lagrangian of Section 2. The cross-section is obtained by folding the amplitudes with 
the luminosities of the $W$ 's and $Z$ 's inside a quark. Both transverse and longitudinal polarizations are included using the distribution functions of Ref. [28] (see also [29]). (The subleading terms in the expressions for these functions depend on the characteristic energy scale of the process under consideration, taken here to be $Q^{2}=m_{Z Z}^{2} / 4$.) The contribution from $W_{L} W_{L}\left(Z_{L} Z_{L}\right)$ fusion, which is the least affected by the choice of $Q^{2}$, is dominant for energies around the peak, since this amplitude is most sensitive to the existence of the Higgs resonance, while the $W_{L} W_{T}+W_{T} W_{T}$ fusion prevails outside this region. The contribution to the cross-section from the interaction of the gauge bosons that does not involve the exchange of the Higgs resonance should in fact be considered as a background [30]. We have calculated this background in the effective theory with $\xi=0$, and subtracted it from the cross-section of the process (21) in our estimates for the signal. We should also remark that in the calculation of both processes (21) and (19), the $s$-channel Higgs boson exchange diagram is unitarized by including the "running" Higgs boson decay width in the propagator. This prescription (which can be justified only in the resonance region) differs from other ones, such as including a constant width, by effects which are formally of higher order in $\lambda \equiv m_{H}^{2} / 2 v^{2}$. However, for $W W$ scattering, it was shown [31] that it is better to use an energy dependent width, because partial wave amplitudes stay closer to the unitarity circle. In terms of event rates, we found that, for gluon fusion, the two prescriptions differ by at most $10 \%$ for a heavy and wide resonance (see also Ref. [32]).

In Tables 10 we present our results for the event rates and the statistical significance of Higgs boson signals for various values of $\xi$ and $y_{t}$. The resonance will have an effective width $\Gamma_{\text {eff }}$ determined by the physical Higgs boson width and by the mass resolution of the detector:

$$
\Gamma_{e f f}=\sqrt{\left(\Delta m_{H}\right)^{2}+\Gamma_{H}^{2}}
$$

We assume that the Higgs boson mass can be resolved to within $\Delta m_{H}=4 \% m_{H}$ [15,21. We have also assumed an identification efficiency of $90 \%$ per lepton [33]. The number of events is measured in a mass bin of width $\Gamma_{\text {eff }}$ centered at the resonance peak. We note that, in all cases examined, this resonance region lies reasonably below the cutoff where the effects of non-unitarity become appreciable. The statistical significance of the signal is determined by computing the Poisson probability that the signal is due to background fluctuations [16. We also note that for large masses and widths the

\footnotetext{
${ }^{3}$ The running width is obtained through the relation $\operatorname{Im} \Pi_{H}(s)=-\sqrt{s} \Gamma_{H}\left(m_{H}^{2}=s\right)$.
} 
resonance peak occurs, in general, at a lower energy than the nominal Higgs boson mass $m_{H}$, due to the interference with the non-resonant terms, the energy dependence of the (running) width and the effect of the falling distribution functions. For example, if $m_{H}=$ 800 (600) $\mathrm{GeV}$ and $\xi=y_{t}=1$, the maximum of the signal cross-section occurs at approximately $730(585) \mathrm{GeV}$. In our results, the Higgs boson mass quoted refers to $m_{H}$ rather than the resonance mass. The results presented for the event rates at the high luminosity $\left(100 \mathrm{fb}^{-1}\right)$ were obtained from those at low luminosity $\left(10 \mathrm{fb}^{-1}\right)$ by scaling by a factor of 10 . This is not, strictly speaking, a correct procedure, because of the problems a higher luminosity environment may pose (such as deterioration in the energy resolution) [16,33. A full detector simulation is needed in order to assess the magnitude of these effects. Consequently, our results for a luminosity of $100 \mathrm{fb}^{-1}$ should be regarded as rather optimistic.

From these tables it can be seen that a nonstandard Higgs resonance may be distinguished in principle from the SM Higgs boson by a comparison of its width and total cross-section to the Standard Model predictions. Before we decide whether this can be achieved in practice, we need to know the expected accuracy of a width measurement, as well as the theoretical uncertainties in the calculation of the width and the cross-section. There are few theoretical uncertainties in the calculation of the SM Higgs boson width. Higher order corrections to both gauge boson and fermion decay modes have been computed [34,35] and have been found to increase the full width by approximately $15 \%$. We chose here not to include this correction, but this does not alter our conclusions. (It will simply change the effective SM value of $\xi$ and $y_{t}$ to a value slightly different from 1.) For the purposes of deciding whether an observed resonance is consistent with the Standard Model predictions, what matters is to know the latter precisely enough, which we do. Similarly, we have chosen not to include radiative corrections to the width of a nonstandard Higgs boson since these can be incorporated into the definition for $\xi$ and $y_{t}$ [6]. In contrast, the accuracy of the cross-section calculation is compromised by the imprecise knowledge of structure functions (amounting to perhaps $30 \%$ for Higgs boson production [16]), our various approximations (such as the effective- $W$ scheme or the neglect of the interference effects of the 'box' diagram in $Z Z$ production) as well as further corrections beyond the included QCD effects. Consequently, if a Higgs-like resonance is discovered, a comparison of its width to the Standard Model prediction offers the best way to probe its nature.

The systematic uncertainty in the measurement of the width arising from smear- 
ing may be corrected for by using eq. (22). This will be an accurate procedure only if $\Gamma_{H} \gtrsim \Delta m_{H}$. The statistical error involved in the measurement of the width warrants a more detailed discussion: Suppose that a Higgs resonance is observed at a mass $m_{H}$ and its width measured and found to differ from the expected Standard Model value $\Gamma_{S M}$. We wish to attach a statistical significance to this deviation. This statistical significance can be derived from the probability density function according to which the possible measurements of the Standard Higgs boson width are distributed. (Any measured quantity is a statistical variable and, as such, obeys some probability distribution function.) To obtain the probability distribution we performed a large number of numerical experiments simulating the possible outcomes of an actual experiment. The procedure adopted was the following: the $Z Z$ invariant-mass range of interest was divided in $4-\mathrm{GeV}$ bins. In each of them the total number of events was generated according to a Poisson distribution with mean $N_{S}+N_{B}$, where $N_{S}, N_{B}$ are the SM signal and background events respectively, expected in that bin. Assuming that the continuum background is known (e.g. from independent experiments) we subtracted the expected background $N_{B}$ in each bin. The resulting distribution represents the signal with an additional noise due to background fluctuations. The mass and the width were obtained by fitting this data with a function of the form

$$
\frac{m^{4}}{E} \frac{e^{-E / E_{0}}}{\left(E^{2}-m^{2}\right)^{2}+m^{2} \Gamma^{2}}
$$

where $E$ is the invariant mass of the $Z$ pair and $m, \Gamma$ are the parameters of the fit. The exponential encodes the effect of the falling parton distribution functions, while in the expression for the cross-section, factors other than the propagator have a rough $\mathrm{m}^{4} / E$ dependence. The value of the constant $E_{0}$ was fixed from the exact (lowest-order) crosssection for the process

$$
p p(g g) \rightarrow H \rightarrow Z Z
$$

The best fit occurs for $E_{0}=283.8 \mathrm{GeV}$.

Repeating this experiment a large number of times, we were able to obtain the probability density, the mean $\left\langle\Gamma_{\text {eff }}\right\rangle$ and the standard deviation $\delta \Gamma_{e f f}$. As mentioned earlier, the physical Higgs boson width can be recovered from the measured, or "effective", width $\Gamma_{\text {eff }}$ through eq. (22). In particular, the spread $\delta \Gamma_{S M}$ that corresponds to one standard 
deviation $\delta \Gamma_{e f f}$ is given by

$$
\delta \Gamma_{S M}=\delta \Gamma_{e f f} \frac{1}{\sqrt{1-\left(\Delta m_{H} / \Gamma_{e f f}\right)^{2}}}
$$

Thus, if a resonance of (physical) width $\Gamma_{H} \neq \Gamma_{S M}$ is observed, the statistical significance $S$ associated with this discrepancy is given by the number of standard deviations that $\Gamma_{H}$ lies away from $\Gamma_{S M}$ :

$$
S=\frac{\left|\Gamma_{H}-\Gamma_{S M}\right|}{\delta \Gamma_{S M}}
$$

Because the underlying statistics is Poisson distributed, we expect that the standard deviation $\delta \Gamma_{\text {eff }}$ scales with the total number of events $N$ like

$$
\frac{\delta \Gamma_{e f f}}{\Gamma_{e f f}}=\frac{c}{\sqrt{N}}
$$

In the limit of large $N$ and negligible background, $c$ is a constant 1 . In general, though, $c$ is a function of both the signal $N$ and the background $B$ (and, as can be expected, increases with increasing $B$ or decreasing $N$ ). For poor statistics and wide objects (for instance in the case $m_{H}=800 \mathrm{GeV}$ ), the width can hardly be measured, even if a statistically significant signal can be obtained.

In Tables 78 we display, for various masses and three representative values of $y_{t}$, namely $y_{t}^{2}=0.5,1$ and 2 , the range of values of $\xi$ for which the nonstandard Higgs boson is observable and distinguishable from the SM Higgs. Results are presented for integrated luminosities of $10 \mathrm{fb}^{-1}$ and $100 \mathrm{fb}^{-1}$. The criteria used in compiling these tables are the following: For a signal to be declared "observable" we require that it consists of at least 10 events and that its statistical significance is greater than $5 \sigma$. For it to be distinguishable from the SM Higgs boson, we require that its width $\Gamma_{H}$ differ from the Standard Model value by at least three standard deviations as defined by eq. (25). If this criterion is not satisfied, one could in principle examine the signal event rate. However, given the large uncertainty in the theoretical calculation, we opted not to use this information.

\footnotetext{
${ }^{4}$ In the narrow width approximation, that is, ignoring the exponential factor $\exp \left(-E / E_{0}\right)$ in eq. (23), we found $c=1.25$. Note that this result is process-independent and reflects only the underlying statistics. It could therefore be applied to other analyses as well.
} 


\section{Conclusions}

Our conclusions are consistent with the expectation that a SM Higgs boson will be detected at the LHC in this channel provided its mass is less than about $500 \mathrm{GeV}$ (at $10 \mathrm{fb}^{-1}$ ) or $800 \mathrm{GeV}$ (at $100 \mathrm{fb}^{-1}$ ). As $y_{t}$ becomes smaller or larger than unity, this mass range will shrink or expand. For example, at $y_{t}^{2}=0.5$ and $\xi=1$ the respective mass ranges at the low and high luminosity options considered are $330 \mathrm{GeV} \lesssim m_{H} \lesssim 430 \mathrm{GeV}$ and $2 M_{Z} \lesssim m_{H} \lesssim 680 \mathrm{GeV}$ respectively. We observe further that at $10 \mathrm{fb}^{-1}$, only models with relatively large $\xi$ can be differentiated from the Standard Model. This is primarily due to the low statistics and the consequent imprecision in the width measurement. It might be possible, however, to improve the statistics by a less strict set of cuts on the final state leptons (or Z's). The situation is considerably better at $100 \mathrm{fb}^{-1}$, as can be seen from Table 8.

In certain cases where $\xi$ is small, the nonstandard Higgs boson is too narrow to be resolved, even though a SM Higgs of the same mass is not. In this case one could tell that the Higgs boson is nonstandard by comparing the detector resolution to the expected SM width, but it is not possible to determine a value for $\xi$.

As we emphasized earlier, the deviation of the values of the parameters $\xi$ and $y_{t}$ from unity is a measure of the cutoff $\Lambda$, which can be thought of as an upper bound to the scale of new physics. Precise relations, however, are model-dependent. In the context of specific models, the results presented in Tables 7-8 reveal the energy scale that the LHC will be able to probe. For example, if $m_{H}=500 \mathrm{GeV}$, where the sensitivity of the LHC to the measurement of $\xi$ is about $30 \%$ (see Table 8), the scale probed is $\Lambda=4.3 \mathrm{TeV}$ in the $S U(3)_{L} \times S U(3)_{R} / S U(3)_{V}$ model of Ref. [8] where $\xi^{2}=1-v^{2} / f^{2}, \Lambda=2.2 \mathrm{TeV}$ in the $S U(5) / S O(5)$ model [9] where $\xi^{2}=1-\left(v^{2} / 4 f^{2}\right)$, and finally $\Lambda=16 \mathrm{TeV}$ in the $S U(4) / S U(2) \times S U(2)$ model 10 in which $\xi^{2}=1-\left(4 v^{2} / f^{2}\right)$. In the above, $\Lambda=4 \pi f$ is the compositeness scale of the underlying new strong dynamics and $v=246 \mathrm{GeV}$, while we have assumed $y_{t}=1$ in all of these cases. In a general Two-Higgs-Doublet model where a gap exists between the mass $m_{H}$ of the lightest neutral state and that of the heavier (nearly degenerate) scalars $(M$, say), the parameter $\xi$ generally approaches its SM value

faster: $\xi^{2}=1-O\left(m_{H}^{4} / M^{4}\right)$; our results indicate that, in this case, it will be very hard to determine the existence of a non-minimal scalar sector solely from a measurement of the width of the observed resonance (see also Ref. [36]). 


\section{Acknowledgements}

We thank R. S. Chivukula, M. Golden, K. Lane and B. Zhou for useful conversations. This work was supported in part under NSF contract PHY-9057173 and DOE contract DE-FG02-91ER40676. 


\begin{tabular}{|c|l|c|l|l|l|c|}
\hline & \multicolumn{2}{|c|}{$m_{H}=200 \mathrm{GeV}$} & \multicolumn{2}{c|}{$m_{H}=350 \mathrm{GeV}$} & \multicolumn{2}{c|}{$m_{H}=500 \mathrm{GeV}$} \\
\hline$\xi$ & Ev. (Sign.) & Width & Ev. (Sign.) & Width & Ev. (Sign.) & Width \\
\hline 0.25 & $39(5.6)$ & 0.09 & $26(7.6)$ & 1.21 & 3.7 & 14.5 \\
\hline 0.50 & $40(5.8)$ & 0.36 & $32(9.0)$ & 4.02 & $11(4.8)$ & 24.2 \\
\hline 0.75 & $42(6.0)$ & 0.80 & $34(9.0)$ & 8.71 & $14(5.2)$ & 40.5 \\
\hline 1.00 & $45(6.4)$ & 1.41 & $36(8.8)$ & 15.3 & $16(5.2)$ & 63.3 \\
\hline 1.25 & $49(6.9)$ & 2.21 & $43(9.3)$ & 23.7 & $18(5.0)$ & 92.5 \\
\hline 1.50 & $54(7.4)$ & 3.18 & $43(8.5)$ & 34.0 & $20(4.6)$ & 128 \\
\hline 1.75 & $59(8.2)$ & 4.32 & $45(8.2)$ & 46.2 & $22(4.4)$ & 170 \\
\hline 2.00 & $66(8.9)$ & 5.65 & $46(7.6)$ & 60.3 & $24(4.0)$ & 219 \\
\hline
\end{tabular}

Table 1: Event rates and decay widths for various Higgs boson masses $m_{H}$ and $\xi$ at the LHC at a luminosity of $10 \mathrm{fb}^{-1}$ for standard top Yukawa coupling and $m_{t}=170 \mathrm{GeV}$. The statistical significance is also shown for signals consisting of more than 10 events.

\begin{tabular}{|c|l|c|l|c|l|l|l|l|c|}
\hline & \multicolumn{2}{|c|}{$m_{H}=200 \mathrm{GeV}$} & \multicolumn{2}{l|}{$m_{H}=400 \mathrm{GeV}$} & \multicolumn{2}{l|}{$m_{H}=600 \mathrm{GeV}$} & \multicolumn{2}{l|}{$m_{H}=800 \mathrm{GeV}$} \\
\hline$\xi$ & Ev. (Sg.) & $\Gamma_{H}$ & Ev. (Sg.) & $\Gamma_{H}$ & Ev. (Sg.) & $\Gamma_{H}$ & Ev. (Sg.) & $\Gamma_{H}$ \\
\hline 0.25 & $389(17.9)$ & 0.09 & $98(12.7)$ & 4.85 & $22(5.5)$ & 25.1 & 6.9 & 48.6 \\
\hline 0.50 & $403(18.5)$ & 0.36 & $205(22.9)$ & 9.40 & $50(9.3)$ & 42.8 & $14(4.0)$ & 92.9 \\
\hline 0.75 & $418(19.0)$ & 0.80 & $264(25.1)$ & 17.0 & $68(10.2)$ & 72.4 & $21(4.2)$ & 167 \\
\hline 1.00 & $446(20.2)$ & 1.41 & $340(28.1)$ & 27.6 & $82(10.1)$ & 114 & $28(4.0)$ & 270 \\
\hline 1.25 & $486(21.8)$ & 2.21 & $352(26.5)$ & 41.2 & $96(9.5)$ & 167 & $36(3.5)$ & 403 \\
\hline 1.50 & $535(23.7)$ & 3.18 & $368(24.6)$ & 57.9 & $111(8.6)$ & 232 & $44(2.8)$ & 565 \\
\hline 1.75 & $593(25.9)$ & 4.32 & $387(22.8)$ & 77.6 & $126(8.0)$ & 309 & \multicolumn{2}{|c|}{$*$} \\
\hline 2.00 & $659(28.4)$ & 5.65 & $406(21.8)$ & 100 & $140(6.4)$ & 398 & \multicolumn{3}{|c|}{$*$} \\
\hline
\end{tabular}

Table 2: Event rates and decay widths for various Higgs boson masses $m_{H}$ and $\xi$ at the LHC at a luminosity of $100 \mathrm{fb}^{-1}$ for standard top Yukawa coupling and $m_{t}=170 \mathrm{GeV}$. The statistical significance is also shown for signals consisting of more than 10 events. The star indicates that the Higgs boson is too wide to be considered a resonance $\left(\Gamma_{H} \sim m_{H}\right)$. 


\begin{tabular}{|c|l|c|l|l|l|c|}
\hline & \multicolumn{2}{|c|}{$m_{H}=200 \mathrm{GeV}$} & \multicolumn{2}{c|}{$m_{H}=350 \mathrm{GeV}$} & \multicolumn{2}{c|}{$m_{H}=500 \mathrm{GeV}$} \\
\hline$\xi$ & Ev. (Sign.) & Width & Ev. (Sign.) & Width & Ev. (Sign.) & Width \\
\hline 0.25 & $20(3.0)$ & 0.09 & $15(4.9)$ & 1.07 & 3.1 & 8.87 \\
\hline 0.50 & $20(3.1)$ & 0.36 & $17(5.4)$ & 3.89 & 6.1 & 18.6 \\
\hline 0.75 & $22(3.3)$ & 0.80 & $18(5.4)$ & 8.57 & 8.8 & 34.9 \\
\hline 1.00 & $25(3.7)$ & 1.41 & $20(5.5)$ & 15.1 & $10(3.5)$ & 57.6 \\
\hline 1.25 & $28(4.2)$ & 2.21 & $25(6.0)$ & 23.6 & $11(3.4)$ & 86.9 \\
\hline 1.50 & $33(4.9)$ & 3.18 & $26(5.7)$ & 33.9 & $13(3.2)$ & 123 \\
\hline 1.75 & $39(5.7)$ & 4.32 & $28(5.6)$ & 46.1 & $14(3.1)$ & 165 \\
\hline 2.00 & $46(6.5)$ & 5.65 & $31(5.3)$ & 60.1 & $16(2.8)$ & 214 \\
\hline \hline SM & $45(6.4)$ & 1.41 & $36(8.8)$ & 15.3 & $16(5.2)$ & 63.3 \\
\hline
\end{tabular}

Table 3: Event rates and decay widths for various masses and $\xi$ at the LHC at a luminosity of $10 \mathrm{fb}^{-1}$ for nonstandard top Yukawa coupling $y_{t}^{2}=0.5$ and $m_{t}=170 \mathrm{GeV}$. The statistical significance is also shown for signals consisting of more than 10 events.

\begin{tabular}{|c|c|c|c|c|c|c|c|c|}
\hline & \multicolumn{2}{|c|}{$m_{H}=200 \mathrm{GeV}$} & \multicolumn{2}{|c|}{$m_{H}=400 \mathrm{GeV}$} & \multicolumn{2}{|c|}{$m_{H}=600 \mathrm{GeV}$} & \multicolumn{2}{|c|}{$m_{H}=800 \mathrm{GeV}$} \\
\hline$\xi$ & Ev. (Sg.) & $\Gamma_{H}$ & Ev. (Sg.) & $\Gamma_{H}$ & Ev. (Sg.) & $\Gamma_{H}$ & Ev. (Sg.) & $\Gamma_{H}$ \\
\hline 0.25 & $198(9.6)$ & 0.09 & $75(10.0)$ & 3.18 & $15(4.1)$ & 15.5 & 5.9 & 31.7 \\
\hline 0.50 & $204(9.9)$ & 0.36 & $126(15.5)$ & 7.73 & $35(7.4)$ & 33.2 & $10(3.0)$ & 76.0 \\
\hline 0.75 & $218(10.5)$ & 0.80 & $152(17.0)$ & 15.3 & $43(7.2)$ & 62.8 & $13(3.0)$ & 150 \\
\hline 1.00 & $245(11.7)$ & 1.41 & $190(18.3)$ & 25.9 & $50(7.0)$ & 104 & $18(2.9)$ & 253 \\
\hline 1.25 & $285(13.5)$ & 2.21 & $202(17.3)$ & 39.5 & $62(6.6)$ & 158 & $25(2.5)$ & 386 \\
\hline 1.50 & $334(15.6)$ & 3.18 & $220(16.6)$ & 56.2 & $74(6.5)$ & 223 & $32(2.2)$ & 549 \\
\hline 1.75 & $392(18.0)$ & 4.32 & $240(15.5)$ & 75.9 & $87(5.7)$ & 299 & $*$ & \\
\hline 2.00 & $458(20.7)$ & 5.65 & $268(15.2)$ & 98.6 & $100(4.7)$ & 388 & * & \\
\hline$\overline{\mathrm{SM}}$ & $\overline{4446(20.2)}$ & 1.41 & $340(28.1)$ & 27.6 & $82(10.1)$ & 114 & $28(4.0)$ & 270 \\
\hline
\end{tabular}

Table 4: Event rates and decay widths for various masses and $\xi$ at the LHC at a luminosity of $100 \mathrm{fb}^{-1}$ for nonstandard Higgs-top Yukawa coupling $y_{t}^{2}=0.5$ and $m_{t}=170 \mathrm{GeV}$. The statistical significance is also shown for signals consisting of more than 10 events. The star indicates that the Higgs boson is too wide to be considered a resonance $\left(\Gamma_{H} \sim m_{H}\right)$. 


\begin{tabular}{|c|l|l|l|l|l|c|}
\hline & \multicolumn{2}{|c|}{$m_{H}=200 \mathrm{GeV}$} & \multicolumn{2}{c|}{$m_{H}=350 \mathrm{GeV}$} & \multicolumn{2}{c|}{$m_{H}=500 \mathrm{GeV}$} \\
\hline$\xi$ & Ev. (Sign.) & Width & Ev. (Sign.) & Width & Ev. (Sign.) & Width \\
\hline 0.25 & $75(9.9)$ & 0.09 & $42(11.1)$ & 1.48 & 4.9 & 25.7 \\
\hline 0.50 & $79(10.5)$ & 0.36 & $59(14.3)$ & 4.29 & $14(5.3)$ & 35.5 \\
\hline 0.75 & $82(10.7)$ & 0.80 & $64(14.5)$ & 8.98 & $21(6.8)$ & 51.7 \\
\hline 1.00 & $85(11.0)$ & 1.41 & $68(14.4)$ & 15.5 & $26(7.1)$ & 74.5 \\
\hline 1.25 & $89(11.5)$ & 2.21 & $78(14.8)$ & 24.0 & $29(7.2)$ & 104 \\
\hline 1.50 & $94(12.0)$ & 3.18 & $75(13.5)$ & 34.3 & $33(6.9)$ & 140 \\
\hline 1.75 & $99(12.6)$ & 4.32 & $77(12.7)$ & 46.5 & $35(6.5)$ & 182 \\
\hline 2.00 & $106(13.3)$ & 5.65 & $76(11.6)$ & 60.5 & $38(5.7)$ & 231 \\
\hline \hline SM & $45(6.4)$ & 1.41 & $36(8.8)$ & 15.3 & $16(5.2)$ & 63.3 \\
\hline
\end{tabular}

Table 5: Event rates and decay widths for various masses and $\xi$ at the LHC at a luminosity of $10 \mathrm{fb}^{-1}$ for nonstandard Higgs-top Yukawa coupling $y_{t}^{2}=2$ and $m_{t}=170 \mathrm{GeV}$. The statistical significance is also shown for signals consisting of more than 10 events.

\begin{tabular}{|c|c|c|c|c|c|c|c|c|}
\hline & \multicolumn{2}{|c|}{$m_{H}=200 \mathrm{GeV}$} & \multicolumn{2}{|c|}{$m_{H}=400 \mathrm{GeV}$} & \multicolumn{2}{|c|}{$m_{H}=600 \mathrm{GeV}$} & \multicolumn{2}{|c|}{$m_{H}=800 \mathrm{GeV}$} \\
\hline$\xi$ & Ev. (Sg.) & $\Gamma_{H}$ & Ev. (Sg.) & $\Gamma_{H}$ & Ev. (Sg.) & $\Gamma_{H}$ & Ev. (Sg.) & $\Gamma_{H}$ \\
\hline 0.25 & $751(31.7)$ & 0.09 & $116(14.6)$ & 8.19 & $23(4.8)$ & 44.2 & 7.6 & 82.4 \\
\hline 0.50 & $795(33.3)$ & 0.36 & $301(28.9)$ & 12.7 & 65( & 61.9 & $20(4.8)$ & 127 \\
\hline 0.75 & 816 & 0.80 & $497(4$ & 20.3 & 100( & 91.5 & 32 & 201 \\
\hline 1.00 & 846( & 1.41 & $546(40$ & $\overline{30.9}$ & 131 & $\overline{133}$ & $44(5)$ & 304 \\
\hline 1.25 & 886( & 2.21 & 611 & 44.5 & 156 & 186 & $56(5.0)$ & 437 \\
\hline 1.50 & 936( & 3.18 & $636(37.4)$ & $\overline{61.2}$ & $178(12.7)$ & 251 & $67(3.9)$ & $\overline{599}$ \\
\hline 1.75 & $995(40.1)$ & 4.32 & $643(34.7)$ & 80.1 & $198(11.1)$ & 328 & & \\
\hline 2.00 & $1060(42.3)$ & 5.65 & $675(33.0)$ & 104 & $214(9.0)$ & 417 & & \\
\hline$\overline{\overline{\text { SM }}}$ & $\bar{~} 446(20.2)$ & $\overline{\overline{1.41}}$ & 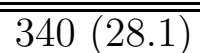 & $\overline{27.6}$ & 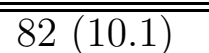 & $\overline{114}$ & $28(4.0)$ & 270 \\
\hline
\end{tabular}

Table 6: Event rates and decay widths for various masses and $\xi$ at the LHC at a luminosity of $100 \mathrm{fb}^{-1}$ for nonstandard Higgs-top Yukawa coupling $y_{t}^{2}=2$ and $m_{t}=170 \mathrm{GeV}$. The statistical significance is also shown for signals consisting of more than 10 events. The star indicates that the Higgs boson is too wide to be considered a resonance $\left(\Gamma_{H} \sim m_{H}\right)$. such an object 


\begin{tabular}{|c|c|c|c|}
\hline \multirow{2}{*}{$m_{H}(\mathrm{GeV})$} & \multicolumn{3}{|c|}{ Range of $\xi$} \\
\cline { 2 - 4 } & $y_{t}^{2}=0.5$ & $y_{t}^{2}=1$ & $y_{t}^{2}=2$ \\
\hline 300 & - & - & - \\
\hline 350 & $\xi \gtrsim 1.70$ & $\xi \gtrsim 1.70$ & $\xi \gtrsim 1.70$ \\
\hline 400 & $1.60 \lesssim \xi \lesssim 1.75$ & $\xi \gtrsim 1.60$ & $\xi \gtrsim 1.55$ \\
\hline 450 & - & $\xi \gtrsim 1.70$ & $\xi \gtrsim 1.65$ \\
\hline 500 & - & - & $\xi \gtrsim 1.80$ \\
\hline
\end{tabular}

Table 7: Range of values of the parameter $\xi$ for which the nonstandard Higgs boson resonance is both observable and distinguishable from the Standard Higgs at $10 \mathrm{fb}^{-1}$ of luminosity and three values of the nonstandard Yukawa coupling $y_{t}$. The range $0<\xi \leq 2.0$ has been explored.

\begin{tabular}{|c|c|c|c|}
\hline$m_{H}(\mathrm{GeV})$ & \multicolumn{3}{|c|}{ Range of $\xi$} \\
\cline { 2 - 4 } & $y_{t}^{2}=0.5$ & $y_{t}^{2}=1$ & $y_{t}^{2}=2$ \\
\hline 200 & - & - & $\xi \gtrsim 1.40$ \\
\hline 300 & $\xi \gtrsim 1.40$ & $\xi \gtrsim 1.40$ & $\xi \gtrsim 1.15$ \\
\hline 400 & $0.20 \lesssim \xi \lesssim 0.45$ & $0.20 \lesssim \xi \lesssim 0.35$ & \\
& $\xi \gtrsim 1.25$ & $\xi \gtrsim 1.20$ & $\xi \lesssim \lesssim{ }^{2}$ \\
\hline 500 & $0.25 \lesssim \xi \lesssim 0.70$ & $0.20 \lesssim \xi \lesssim 0.60$ & $0.20 \lesssim \xi \lesssim 0.40$ \\
& $\xi \gtrsim 1.35$ & $\xi \gtrsim 1.30$ & $\xi \gtrsim 1.20$ \\
\hline 600 & $\xi \gtrsim 1.45$ & $\xi \gtrsim 1.40$ & $\xi \gtrsim 1.35$ \\
\hline 700 & - & $1.60 \lesssim \xi \lesssim 1.90$ & $\xi \gtrsim 1.55$ \\
\hline 800 & - & - & - \\
\hline
\end{tabular}

Table 8: Range of values of the parameter $\xi$ for which the nonstandard Higgs boson resonance is both observable and distinguishable from the Standard Higgs at $100 \mathrm{fb}^{-1}$ of luminosity and three values of the nonstandard Yukawa coupling $y_{t}$. The range $0<\xi \leq 2.0$ has been explored. 


\section{References}

[1] ALEPH Collaboration, D. Decamp et al., Phys. Rev. 216 (1992) 253;

DELPHI Collaboration, P. Abreu et al., Nucl. Phys. B373 (1992) 3;

L3 Collaboration, O. Adriani et al., Phys. Lett. B303 (1993) 391;

OPAL Collaboration, M. Akrawy et al., Phys. Lett. B253 (1991) 511.

[2] R. Dashen and H. Neuberger, Phys. Rev. Lett. 50 (1983) 1897.

[3] B.W. Lee, C. Quigg and H. Thacker, Phys. Rev. D16 (1977) 1519.

[4] G.'t Hooft, in: Recent Developments in Gauge Theories, edited by G. 't Hooft (Plenum Press, New York, 1980).

[5] R. S. Chivukula and V. Koulovassilopoulos, Phys. Lett. B309 (1993) 371.

[6] V. Koulovassilopoulos and R. S. Chivukula, Phys. Rev. D50 (1994) 3218.

[7] D. B. Kaplan and H. Georgi, Phys. Lett. B136 (1984) 183;

D. B. Kaplan, S. Dimopoulos and H. Georgi, Phys. Lett. B136 (1984) 187.

[8] T. Banks, Nucl. Phys. B243 (1984) 125.

[9] H. Georgi and D. B. Kaplan, Phys. Lett. B145 (1984) 216;

M. J. Dugan, H. Georgi and D. B. Kaplan, Nucl. Phys. B254 (1985) 299.

[10] D. A. Kosower, in Physics of the Superconducting Supercollider, Proceedings of the 1986 Summer Study on the Physics of the SSC, Snowmass 1986, edited by R. Donaldson and J. Marx.

[11] Y. Nambu, Enrico Fermi Institute Preprint EFI 88-39;

V. A. Miransky, M. Tanabashi, and K. Yamawaki, Phys. Lett. B221 (1989) 177;

Mod. Phys. Lett. A4 (1989) 1043;

W. A. Bardeen, C. T. Hill, and M. Lindner, Phys. Rev. D41 (1990) 1647.

[12] S. Coleman, J. Wess and B. Zumino, Phys. Rev. 177 (1969) 2239;

C. G. Callan, S. Coleman, J. Wess and B. Zumino, Phys. Rev. 177 (1969) 2247.

[13] R. S. Chivukula, M. Dugan and M. Golden, Phys. Lett. B336 (1994) 62. 
[14] S. Weinberg, Physica 96 A (1979) 327;

J. Gasser and H. Leutwyler, Ann. Phys. (N.Y.) 158 (1984) 142; Nucl. Phys. B250 (1985) 465.

[15] D. Froidevaux, in Proceedings of the ECFA Large Hadron Collider Workshop, Aachen 1990, (G. Jarlskog and D. Rein, eds.), Vol. II, p. 444, and references therein.

[16] GEM Technical Design Report; GEM TN-93-262, SSCL-SR-1219; Submitted by the GEM Collaboration to the Superconducting Supercollider Laboratory (April 30, 1993).

[17] A. Rubbia, talk presented at the Conference Beyond the Standard Model IV, Lake Tahoe, California, December 13-18, 1994.

[18] See, for example E. J. Eichten, C. Quigg, I. Hinchliffe and K. D. Lane, Rev. Mod. Phys. 56 (1984) 579.

[19] V. Barger, J. L. Lopez and W. Putikka, Int. J. Mod. Phys. A3 (1988) 2181.

[20] E. W. N. Glover and J. J. van der Bij, Nucl. Phys. B321 (1989) 561;

U. Baur and E. W. N. Glover, in Proceedings of the ECFA Large Hadron Collider Workshop, Aachen 1990, (G. Jarlskog and D. Rein, eds.), Vol. II, p. 570;

U. Baur and E. W. N. Glover, Phys. Rev. D44 (1991) 99.

[21] A. Nisati, in Proceedings of the ECFA Large Hadron Collider Workshop, Aachen 1990, (G. Jarlskog and D. Rein, eds.), Vol. II, p. 492.

[22] CDF Collaboration, F. Abe et al., Fermilab-Pub-94/0097-E.

[23] A. Djouadi, M. Spira and P. Zerwas, Phys. Lett. B264 (1991) 440.

[24] D. Graudenz, M. Spira and P. Zerwas, Phys. Rev. Lett. 70 (1993) 1372.

[25] Z. Kunszt and F. Zwirner, Nucl. Phys. B385 (1992) 3.

[26] M. Chanowitz and M. K. Gaillard, Phys. Lett. B142 (1984) 85;

G. Kane, W. Repko and W. Rolnik, Phys. Lett. B148 (1984) 367;

S. Dawson, Nucl. Phys. B249 (1985) 42.

[27] M. Chanowitz and M. K. Gaillard, Nucl. Phys. B261 (1985) 379. 
[28] A. Abbasabadi and W. W. Repko, Phys. Rev. D36 (1987) 289.

[29] U. Baur and E. W. N. Glover, Nucl. Phys. B347 (1990) 12.

[30] J. Bagger, V. Barger, K. Cheung, J. Gunion, T. Han, G. A. Ladinsky, R. Rosenfeld and C.-P. Yuan, Phys. Rev. D49 (1994) 1246.

[31] G. Valencia and S. D. Willenbrock, Phys. Rev. D46 (1992) 2247;

S. D. Willenbrock and G. Valencia, Phys. Lett. B247 (1990) 341.

[32] E. W. N. Glover, in High Energy Hadronic Interactions '91, Proceedings of the 26th Rencontre de Moriond, Les Arcs, Savoie, France, edited by J. Tran Thanh Van (Editions Frontieres, Gif-sur-Yvette, 1991), p. 161.

[33] C. Seez et al., in Proceedings of the ECFA Large Hadron Collider Workshop, Aachen 1990, (G. Jarlskog and D. Rein, eds.), Vol. II, p. 474.

[34] J. Fleischer and F. Jegerlehner, Phys. Rev. D23 (1981) 2001;

N. Sakai, Phys. Rev. D22 (1980) 2220;

B. A. Kniehl, Nucl. Phys. B357 (1991) 439;

S. G. Gorishny, A. L. Kataev, S. A. Larin and L. R. Surguladze, Mod. Phys. Lett. A5 (1990) 2703;

B. A. Kniehl, Phys. Rev. 240 (1994) 211.

[35] E. Braaten and J. Leveille, Phys. Rev. D22 (1980) 715;

M. Drees and K. Hikasa, Phys. Lett. B240 (1990) 445; 262 (1991) 497;

R. Kleiss, Z. Kunszt and W. J. Stirling, Phys. Lett. B253 (1991) 269.

[36] H. E. Haber, preprint SCIPP 94/39, hep-ph 9501320. 